\title{
Encapsulation of Streptococcus Salivarius in Double Emulsion Droplets as a Method for Increasing the Efficacy of Oral Topical Medications
}

\author{
Seung Ah Choi ${ }^{1}$ and Soonmin Lee ${ }^{2,3 *}$ \\ ${ }^{1}$ Monta Vista High School, Cupertino, CA, US \\ ${ }^{2}$ Department of Pediatrics, Yonsei University, College of Medicine, Seoul, Korea \\ ${ }^{3}$ Department of Pediatrics, Division of Neonatal \& Developmental Medicine, Stanford, CA, US
}

*Corresponding author: Soonmin Lee, Department of Pediatrics, Gangnam Severance Hospital, Yonsei University College of

Medicine, 211 Eonjuro, Gangnam-gu, Seoul 06273, Republic of Korea.

\section{ARTICLE INFO}

Received: 幽 June 21, 2019

Published: June 28, 2019

Citation: Seung Ah Choi, Soon Min Lee. Encapsulation of Streptococcus Salivarius in Double Emulsion Droplets as a Method for Increasing the Efficacy of Oral Topical Medications. Biomed J Sci \& Tech Res 19(2)-2019. BJSTR. MS.ID.003271.

Abbreviations: DED: Double Emulsion Droplets

\begin{abstract}
Streptococcus alivarius, a flora present in the human oral cavity, plays an important role in wound healing. This study aimed to optimize double emulsion droplets (DEDs) to encapsulate Streptococcus Salivarius with increased viscosity and adhesiveness as an oral topical medication. Streptococcus Salivarius grew nearly as well in saliva (68\%) as in broth and grew significantly better than the control $(\mathrm{p}<0.05)$ over 72 hour. The diameter of DED according to 10 weight $\%$ PVA and 15 weight \% n-hexadecane span 80 was the largest. The percentage of the ointment absorbed into the oral epithelium was $88.44 \%$ of DEDs for optimal $80 \%$ formulation compared to $64.63 \%$ the control containing $0 \%$ DEDs. Double emulsion droplets that encapsulated S. Salivarius increased the efficacy of the topical medication in the oral cavity and improved wound healing in the oral cavity.
\end{abstract}

Keywords: Streptococcus Salivarius; Double Emulsion Droplets; Topical Medication; Oral Cavity

\section{Introduction}

Good oral health is critical for general health and well-being. Several innovative drug delivery systems have been developed for local treatment of various oral diseases [1]. However, these delivery systems exhibit limited efficacy and retention at the target site [2]. Bio-adhesive formulations may be a potential mechanism to improve drug delivery to the oral cavity. Probiotics enhance mucosal barrier function and promote intestinal epithelial survival and growth [3-5]. Probiotic bacteria in the skin and in the oral cavity have received increasing attention [6,7]. Recent studies have evaluated the antimicrobial activity of Lactobacillus, and the ability of Lactobacillus to promote host-microbe homeostasis [810]. Streptococcus Salivarius is one of the first colonizers of the human oral cavity. It plays an important role in wound healing and anti-inflammatory processes in human intestinal epithelial cells [11]. Several strains of $S$. Salivarius have been shown to stimulate re-epithelialization in the gingiva. Further analysis showed that
S. Salivarius significantly promoted re-epithelialization through a secreted proteinaceous compound. S. Salivarius also was attempted to use as probiotics to prevent fungal infection.

Water-in-oil-in-water double emulsions are dynamic systems with potential applications in many fields. These emulsions are characterized by water droplets dispersed within oil droplets, which are then dispersed within a secondary water phase. These emulsions have high encapsulation potential, and represent a simple, low-cost method to increase viscosity. Double emulsions also can effectively mask undesirable tastes. Previous studies have demonstrated encapsulation of iron or milk fat within double emulsions [7-11]. Encapsulation of S. Salivarius using double emulsions may be a potential oral therapeutic strategy. These double emulsions may allow for the targeting of specific wounds using hydrophilic properties and may improve the taste and texture of current medications [12]. Furthermore, double emulsions may improve drug absorption and adhesion. This study 
aimed to optimize double emulsion droplets (DED) to encapsulate S. Salivarius. We confirmed that the optimized DED had increased viscosity and adhesiveness in the oral cavity.

\section{Materials and Methods}

To determine the optimal base for cultivation of S. Salivarius, $10 \mathrm{ml}$ of sterile tryptic soy agar with 5\% sheep blood agar (Becton Dickinson $\mathrm{GmbH}$, Heidelberg, Germany), $10 \mathrm{ml}$ of saliva, and $10 \mathrm{ml}$ of distilled water were added to separate petri dishes [13]. One vial of S. Salivarius (Carolina Biological Supply Company, Burlington, USA) was added to each petri dish, then incubated for $4 \mathrm{~h}$ at $37^{\circ} \mathrm{C}$. One colony from each of the petri dishes was placed in a new petri dish. Ten milligrams of acid dye PI (Biotium, Inc., Fremont, USA) was added in each petri dish. To determine the best conditions for DED stability, 1 vial of S. Salivarius was added to distilled water with differing amounts of PVA and n-hexadecane [14]. The percentage of each solute in water was reported as wt $\%$.

To produce the DED, a cylindrical capillary with an inner diameter of $0.58 \mathrm{~mm}$ and a square capillary with an inner diameter of $1.05 \mathrm{~mm}$ were used. A gastight syringe was put at the opening of the cylindrical capillary to narrow the opening. The condition was at room temperature $\left(25^{\circ} \mathrm{C}\right)$ to form the water droplets. Each vial of $S$. salivarius was used to produce approximately 100 DEDs. To determine the optimal amount of DED required to absorb the ointment, the inner mucosa ( $5 \mathrm{~mm} * 5 \mathrm{~mm}$ ) from pig oral cavity was extracted and prepared. A sterile swab was used to apply $2 \mathrm{~g}$ of triamcinolone ointment (Dongkook Pharmaceutical, Seoul, Korea). The amount of DED mixed in the ointment ranged from $0 \%$ to $95 \%(0 \%, 25 \%, 50 \%, 75 \%, 80 \%, 95 \%)$ of the total amount of ointment with DED. Ten milliliters of water were flushed into each pig oral cavity with the ointment using a plank angled at $15^{\circ} \mathrm{C}$ to ensure similar rates of water flow. The water that flushed off of the ointment was collected in a beaker. The liquid collected in the beaker was weighed. The absorbance of the solution collected in the beaker was measured at $620 \mathrm{~nm}$ (S. Salivarius) [15] and 100 $\mathrm{nm}$ (biological tissue) using a spectrophotometer. The percentage of the ointment absorbed into the oral epithelium was calculated as the length of the epithelium absorbed over the length original multiplied by 100 . Since the cross-section of the epithelium is very small, the length of the epithelium tissue from the jaw was pulled out and measured instead of measuring the total area absorbed.

The case of $80 \%$ DED from above was used as the amount of DED to include in the ointment $\left(25 \mathrm{~cm}^{2}\right)$ to be applied to the pig jaw. Water was added to cover the ointment. The length of the ointment penetrated into the tissue was extracted from the tissue and measured after $1 \mathrm{~h}$. Comparisons of between tryptic soy broth, saliva, and the control group were performed using chi-squared test for categorical data. All statistical analyses were performed using SPSS software version 25.0 (Armonk, NY: IBM Corp) and P values of $<0.05$ were considered statistically significant. This study was approved by the review board of Synopsys Santa Clara Valley Science and Engineering Fair Association.

\section{Results}

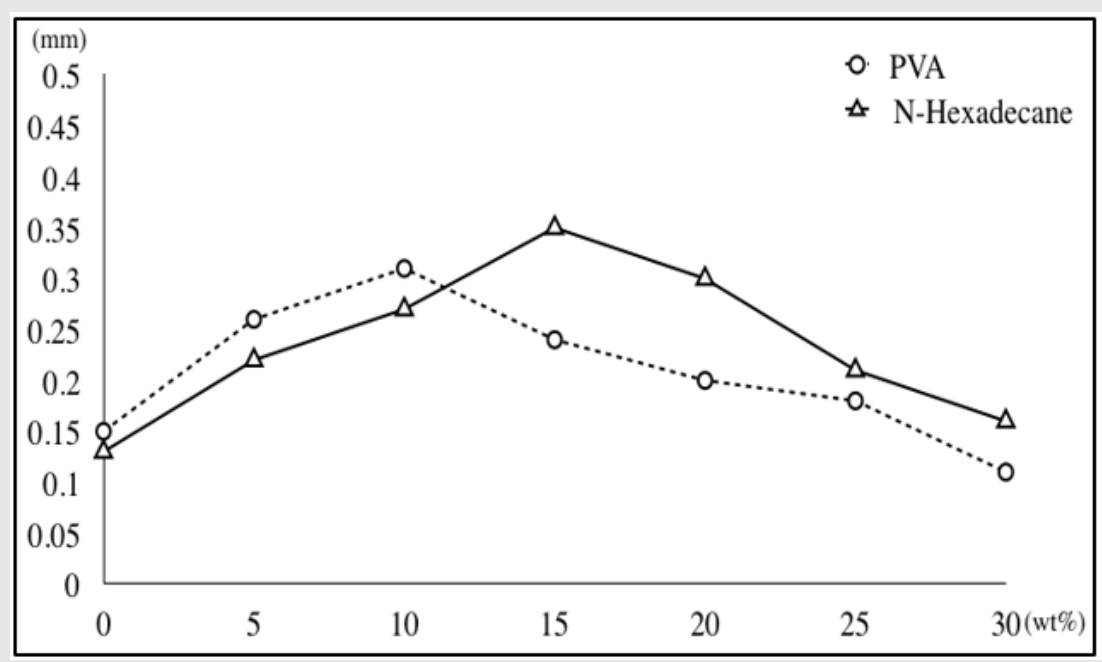

Figure 1: Diameter of double emulsion droplets according to the weight percent of PVA and N-hexadecane.

Tryptic soy broth (79\%) resulted in the best growth. However, growth in saliva $(68 \%)$ was very similar to that in broth $(\mathrm{p}=0.078)$ and was significantly better than that in the control group (42\%) $(\mathrm{p}<0.05)$ after $72 \mathrm{~h}$. (Figure 1) shows the diameters of DEDs based on composition of PVA and n-hexadecane span 80 . The formulation consisting of $10 \mathrm{wt} \% \mathrm{PVA}$ and $15 \mathrm{wt} \%$ n-hexadecane span 80 resulted in the most stable DED. The size of the DED with 0 wt\%
PVA and $0 \mathrm{wt} \% \mathrm{n}$-hexadecane was $0.12 \pm 0.04(\mathrm{~mm})$ and the size of the DED with $10 \mathrm{wt} \%$ PVA and $15 \mathrm{wt} \% \mathrm{n}$-hexadecane was $0.31 \pm$ $0.06(\mathrm{~mm})$. The mass $(\mathrm{g})$ collected in the beaker was between 11$12 \mathrm{~g}$ across the different percentages of DEDs in the ointments. The mass collected was lowest at $80 \%$, suggesting that most of the DEDs adhered to the pig jaw skin under this condition Figure 2 shows the masses that failed to adhere to the pig jaw under each condition 
Figure 3 shows the spectrophotometric results used to confirm the composition of the tissues. The results showed that $16-27 \%$ was biologic tissue and $3-12 \%$ was S. Salivarius. The $80 \%$ ointment formulation had the lowest percentage of S. Salivarius (3.1\%). The length of the ointment absorbed into the oral epithelium showed $0.26 \pm 0.09 \mathrm{~mm}$ of $80 \%$ DED compared to $0.19 \pm 0.07 \mathrm{~mm}$ of control. The percentage of ointment absorbed into the oral epithelium was $88.44 \%$ for the $80 \%$ formulation compared to $64.63 \%$ for the control formulation that contained $0 \%$ DEDs. The percentage of DEDs plotted against the effective time (hours) it lasts is shown in Figure 4 . Almost $54 \%$ of DEDs were stable for more than 24 hour. Addition of $0.4 \%$ salt to the saliva and $10 \mathrm{wt} \%$ PVA to the oil increased the percentage of DEDs that lasted for more than 24 hour from $9 \%$ to $54 \%$.

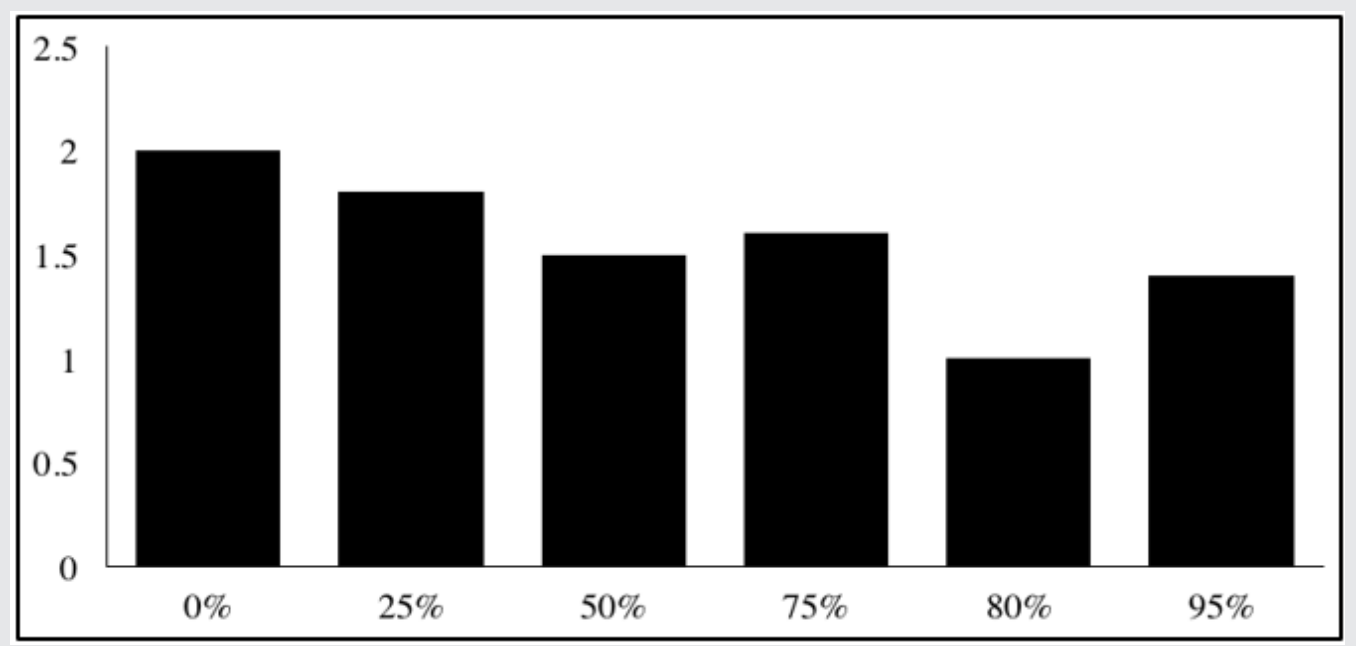

Figure 2: Mass of Substance that failed to adhere to the jaw.

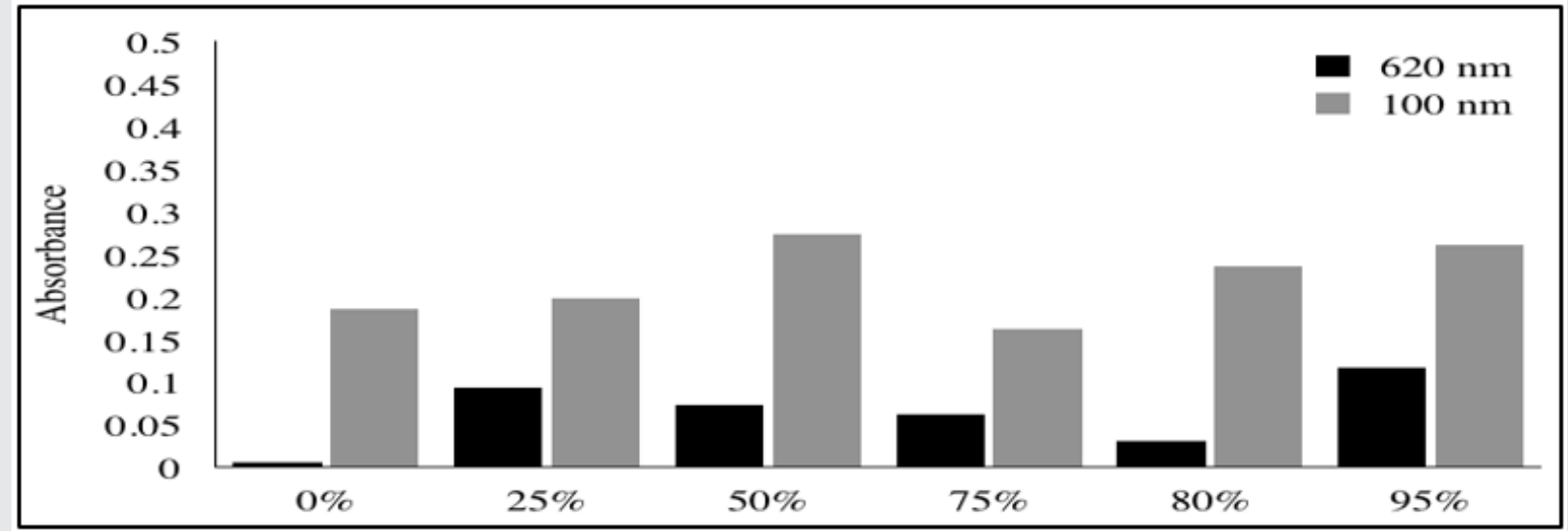

Figure 3: Absorbance for each case at $620 \mathrm{~nm}$ and $100 \mathrm{~nm}$.

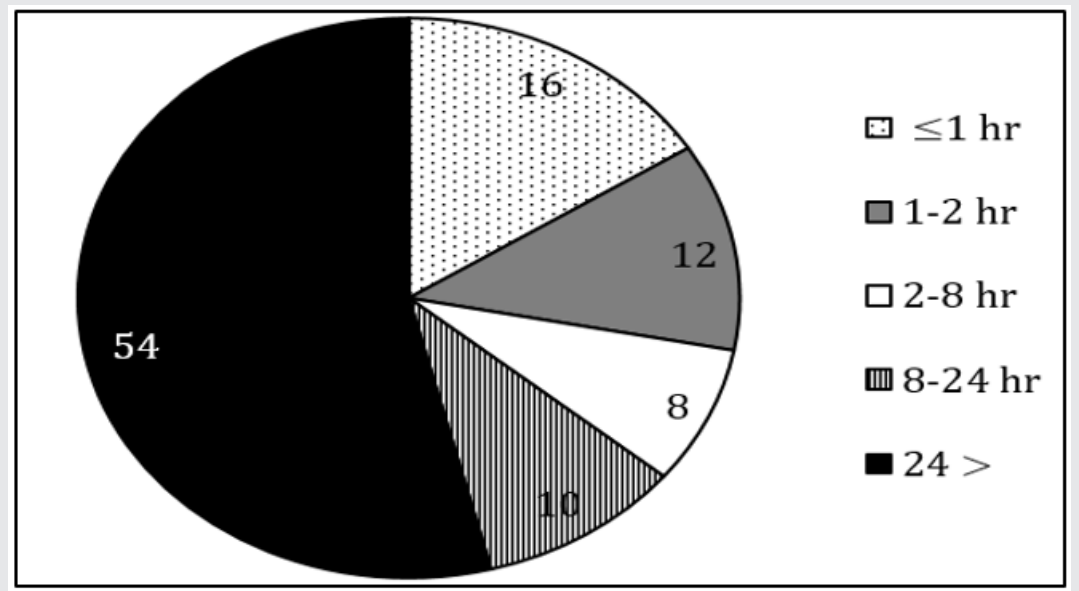

Figure 4: Distribution of double emulsion droplets in percentage depending on the time it lasts. 


\section{Discussion}

Topical medication for use in the oral cavity can be difficult to apply and may not adhere to the wound site due to increased saliva and mucosal irritability [16]. This was the first study to use the double emulsion technique for encapsulating S. Salivarius has been shown to speed the healing process, and DEDs can act as carriers for S. Salivarius, and improve the viscosity of the ointment. Determination of optimal conditions for producing double emulsion droplets that encapsulate $S$. Salivarius resulted in a promising drug delivery system for use in the oral mucosa. $S$. Salivarius is the predominant colonizer of oral mucosal surfaces in humans and does not initiate infections in healthy individuals [5]. In adult saliva samples, levels of S. Salivarius ranged from 106 to 107 colony forming units (CFU)/ml [17].

We confirmed that human saliva can be a good cultivation base for S. Salivarius by comparing the results with the recommended broth and a control. S. Salivarius probiotics are used to control diverse bacterial infections. S. Salivarius K12 can be detected at the mucosal membranes for as long as 3 weeks, but the numbers steadily decrease after day 8 [18]. Wound healing and re-epithelization are a set of complex processes that are initiated upon tissue injury [19]. However, there are many barriers to wound healing in the oral cavity [20]. As such, mechanisms to protect medications in the oral cavity are critical. Development of DEDs that encapsulate S. Salivarius may be an option to improve drug delivery to the oral cavity. This study identified the optimal temperature and solvent volumes to produce the largest DEDs. Use of $10 \mathrm{wt} \%$ PVA and $15 \mathrm{wt} \% \mathrm{n}$-hexadecane span 80 resulted in the most stable DEDs. These results agreed with another study that showed 10\% PVA/hexadecane containing $2 \%$ Span 80/10\% PVA - ultrathin DED was the most effective.

Drug efficacy is influenced by the serum concentrations of drug [21]. However, sampling is invasive. This study evaluated the efficacy of a topical drug using viscosity as an indicator and determined what percent of DEDs should be included in the ointment for maximum adherence and absorption.Determination of stability is important for commercialization [22]. DEDs used to encapsulate S. Salivarius were stable for $72 \mathrm{~h}$. However, making water-in-oilin-water emulsion droplets containing $S$. Salivarius by melting the ointment to mix in the oil base, $50 \%$ of DED with topical medication was stable during 24 hour, it is necessary to make staying longer in further study. A limitation of this study was only a study using an animal model. In addition, the exact amount of $S$. Salivarius included in the DEDs was not calculated exactly. DEDs are applied on the skin as an ointment, not in a completely mixed state with DED. To improve the effective time of DEDs in the oral cavity, more work will need to be performed. In addition, due to experimental constraints, we only indirectly tested the effectiveness of this novel formulation for wound healing, adhesion, and absorbance. Only $620 \mathrm{~nm}$ and $100 \mathrm{~nm}$ was used as a cut off, further study is needed. To design a more efficient topical medication, this study proposed a simpler and more stable formulation generated by combining DEDs and S. Salivarius. This formulation is unique because it is the first to use DEDs for topical application. Human clinical trials are needed to further evaluate the safety and efficacy of these formulations.

\section{Conclusion}

DEDs with encapsulated S. Salivarius increased the efficacy of a topical medication in the oral cavity. This may be a promising formulation to more effectively promote wound healing in the oral cavity.

\section{References}

1. Politis C, Schoenaers J, Jacobs R, Agbaje JO (2016) Wound healing problems in the mouth. Front Physiol 7(507): 1-13.

2. Nguyen S, Hiorth M (2015) Advanced drug delivery systems for local treatment of the oral cavity. Ther Deliv 6(5): 595-608.

3. Anderson RC, Cookson AL, Mc Nabb WC, Kelly WJ, Roy NC (2010) Lactobacillus plantarum dsm 2648 is a potential probiotic that enhances intestinal barrier function. FEMS Microbiol Lett 309(2): 184-192.

4. Johnson Henry KC, Donato KA, Shen Tu G, Gordanpour M, Sherman PM (2008) Lactobacillus rhamnosus strain gg prevents enterohemorrhagic escherichia coli o157:H7-induced changes in epithelial barrier function. Infect Immun 76(4): 1340-1348.

5. Karczewski J, Troost FJ, Konings I, Dekker J, Kleerebezem M, et al. (2010) Regulation of human epithelial tight junction proteins by lactobacillus plantarum in vivo and protective effects on the epithelial barrier. Am J Physiol Gastrointest Liver Physiol 298(6): 851-859.

6. Mohammedsaeed W, Cruickshank S, McBain AJ, O’Neill CA (2015) Lactobacillus Rhamnosus gg lysate increases re-epithelialization of keratinocyte scratch assays by promoting migration. Sci Rep 5(16147): $1-11$.

7. Gungor OE, Kirzioglu Z, Kivanc M (2015) Probiotics: Can they be used to improve oral health? Benef Microbes. 6(5): 647-656.

8. Sookkhee S, Chulasiri M, Prachyabrued W (2001) Lactic acid bacteria from healthy oral cavity of thai volunteers: Inhibition of oral pathogens. J Appl Microbiol 90(2):172-179.

9. Terai T, Okumura T, Imai S, Nakao M, Yamaji K, et al. (2015) Screening of probiotic candidates in human oral bacteria for the prevention of dental disease. PLos One. 10(6): 1-20.

10. Cosseau C, Devine DA, Dullaghan E, Gardy JL, Chikatamarla A, et al. (2008) The commensal streptococcus Salivarius k12 downregulates the innate immune responses of human epithelial cells and promotes hostmicrobe homeostasis. Infect Immun 76(9): 4163-4175.

11. Fernandez Gutierrez MM, Roosjen PPJ, Ultee E, Agelink M, Vervoort JJM, et al. (2017) Streptococcus Salivarius ms-oral-d6 promotes gingival reepithelialization in vitro through a secreted serine protease. Sci Rep. 7(11100): 1-15.

12. Dickinson GME. (2017) Double emulsions relevant to food systems: Preparation, stability, and applications. Comprehensive Reviews in Food Science and Food Safety 16(3): 532-555.

13. Roger P, Delettre J, Bouix M, Beal C (2011) Characterization of streptococcus salivarius growth and maintenance in artificial saliva. J Appl Microbiol 111(3): 631-641.

14.Zhao CX, Chen D, Hui Y, Weitz DA, Middelberg APJ (2017) Controlled generation of ultrathin-shell double emulsions and studies on their stability. Chemphyschem 18(10): 1393-1399.

15. Ishijima SA, Hayama K, Burton JP, Reid G, Okada M, et al. (2012) Effect of Streptococcus Salivarius k12 on the in vitro growth of candida albicans and its protective effect in an oral candidiasis model. Appl Environ Microbiol. 78(7): 2190-2199.

16. Brand HS, Ligtenberg AJ, Veerman EC (2014) Saliva and wound healing. 


\section{Monogr Oral Sci 24: 52-60.}

17. Wescombe PA, Hale JD, Heng NC, Tagg JR (2012) Developing oral probiotics from streptococcus salivarius. Future Microbiol 7(12): 13551371.

18. Burton JP, Wescombe PA, Moore CJ, Chilcott CN, Tagg JR (2006) Safety assessment of the oral cavity probiotic streptococcus salivarius k12. Appl Environ Microbiol 72(4): 3050-3053.

19. Pastar I, Stojadinovic O, Yin NC, Ramirez H, Nusbaum AG, et al. (2014) Epithelialization in wound healing: A comprehensive review. Adv Wound Care 3(7): 445-464.

\section{ISSN: 2574-1241}

DOI: 10.26717/BJSTR.2019.19.003271

Soon Min Lee. Biomed J Sci \& Tech Res

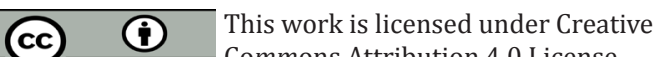

Submission Link: https://biomedres.us/submit-manuscript.php
20. Politis C, Schoenaers J, Jacobs R (2016) Wound healing problems in the mouth. Front Physiol 7(507): 1-13.

21. Ohning BL (1995) Neonatal pharmacodynamics--basic principles. I: Drug delivery. Neonatal Netw 14(2): 7-12.

22. Bajaj S, Singla D, Sakhuja N (2012) Stability testing of pharmaceutical products. J App Pharm Sci 02(03): 129-38.

$\begin{array}{ll}\text { BIOMEDICAL } & \text { Assets of Publishing with us } \\ \text { RESEARCHES } & \text { - Global archiving of articles } \\ & \text { - Immediate, unrestricted online access } \\ & \text { - Rigorous Peer Review Process } \\ & \text { - Authors Retain Copyrights }\end{array}$

